\title{
Dominated chain recurrent class with singularities
}

\author{
Christian Bonatti, ShaObo GAN AND DAWEi YANG
}

\begin{abstract}
We prove that for $C^{1}$ generic three-dimensional vector fields, dominated chain recurrent classes with singularities and periodic orbits are singular hyperbolic.
\end{abstract}

Mathematics Subject Classification (2010): 37C10 (primary); 37D30 (secondary).

\section{Introduction}

Hyperbolicity has been introduced by Smale [24] for understanding chaotic dynamical behavior and it remains a very important concept for understanding even nonhyperbolic systems. For flows on 3-manifolds, Lorenz attractor has been the first discovered robustly non hyperbolic systems, first announced by using numerical experiment [13], and then rigorously modeled in $[1,9,10]$ by the so called geometric model of Lorenz attractor. Lorenz-like attractors share many properties with hyperbolic attractors but involve singular and non-singular orbits in a same transitive dynamics.

In [17] Morales, Pacifico and Pujals introduced singular hyperbolicity to formalize the hyperbolic properties of Lorenz attractors and their generalizations, including the singularities it contains.

The aim of this paper is to give a $C^{1}$-generic characterization of the singular hyperbolicity by a weaker notion called dominated splitting. In order to state precisely our results, we need some definitions.

\subsection{Definitions: singular hyperbolicity, partial hyperbolicity and dominated splittings}

Given a closed Riemannian manifold $M$, we denote by $\mathcal{X}^{r}(M)$ the space of the $C^{r}$ vector fields on $M$ with the usual $C^{r}$ norm.

D. Yang thanks the support of NSFC 11001101 and Ministry of Education of P. R. China 20100061120098 and 200903279. S. Gan is supported by 973 program $2011 \mathrm{CB} 808002$ and NSFC 11025101.

Received June 18, 2012; accepted November 12, 2012. 
Given a vector field $X \in \mathcal{X}^{1}(M)$, we denote its flow by $\phi_{t}^{X}$ and its tangent flow by $\Phi_{t}^{X}=d \phi_{t}^{X}$. If there is no confusion, we will use $\phi_{t}$ and $\Phi_{t}$ for simplicity. We denote by $\operatorname{Sing}(X)=\{x \in M: X(x)=0\}$ the set of singularities of $X$. If a subset $\Lambda \subset M \backslash \operatorname{Sing}(X)$, we say that $\Lambda$ is non-singular.

One says that a compact invariant set $\Lambda$ has a dominated splitting if there is a continuous invariant splitting $T_{\Lambda} M=E \oplus F$, and two constants $C \geq 1$ and $\lambda>0$ such that for any $x \in \Lambda$ and $t \geq 0$, one has

$$
\left\|\left.\Phi_{t}\right|_{E(x)}\right\|\left\|\left.\Phi_{-t}\right|_{F\left(\phi_{t}(x)\right)}\right\| \leq C \mathrm{e}^{-\lambda t} .
$$

If $\operatorname{dim} E$ is a constant, then $\operatorname{dim} E$ is called the index of the dominated splitting.

An invariant bundle $E \subset T_{\Lambda} M$ is called contracting if there are two constants $C \geq 1$ and $\lambda>0$ such that for any $x \in \Lambda$ and $t \geq 0$, one has $\left\|\left.\Phi_{t}\right|_{E(x)}\right\| \leq C \mathrm{e}^{-\lambda t}$. An invariant bundle $F$ is called expanding if it is contracting for the flow generated by $-X$. If $T_{\Lambda} M=E \oplus F$ is a dominated splitting and either $E$ is contracting or $F$ is expanding, then one says that $\Lambda$ is partially hyperbolic.

A continuous invariant bundle $E \subset T_{\Lambda} M$ is called sectional contracting if there are two constants $C \geq 1$ and $\lambda>0$ such that for any $x \in \Lambda, t \geq 0$ and any two-dimensional subspace $L \subset E(x)$, one has $\left|\operatorname{Det}\left(\left.\Phi_{t}\right|_{L}\right)\right| \leq C \mathrm{e}^{-\lambda t}$. A continuous invariant bundle $F$ is called sectional expanding if it is sectional contracting for $-X$.

A compact invariant set $\Lambda$ is called singular hyperbolic if $\Lambda$ has a partially hyperbolic splitting $T_{\Lambda} M=E \oplus F$, and either $E$ is sectional contracting and $F$ is expanding, or $E$ is contracting and $F$ is sectional expanding. For instance, the geometrical Lorenz attractor is singular hyperbolic, but not hyperbolic (see [2]).

Here a compact invariant set $\Lambda$ is called hyperbolic if there is a continuous invariant splitting $T_{\Lambda} M=E^{s} \oplus\langle X\rangle \oplus E^{u}$, where $E^{s}$ is uniformly contracting, $E^{u}$ is uniformly expanding, and $\langle X\rangle$ is the subspace generated by the vector field. If $\operatorname{dim} E^{s}$ is constant, then $\operatorname{dim} E^{s}$ is called the index (or stable index) of the hyperbolic set $\Lambda$.

The dynamical object we will consider are compact invariant sets satisfying some recurrence. The most general setting is the chain recurrence defined by Conley by considering pseudo-orbits; let us recall these notions.

Given $x, y \in M, \varepsilon>0$, we say $\left\{\left(x_{0}, t_{0}\right),\left(x_{1}, t_{1}\right), \cdots,\left(x_{n}, t_{n}\right)\right\}$ is an $\varepsilon$-pseudoorbit (or $\varepsilon$-chain) from $x$ to $y$ if

- $x_{0}=x$ and $x_{n}=y$,

- $t_{i} \in[1,2]$ for each $i$,

- $d\left(\phi_{t_{i}}\left(x_{i}\right), x_{i+1}\right)<\varepsilon$ for any $0 \leq i \leq n-1$.

If for any $\varepsilon>0$, there exists an $\varepsilon$-chain from $x$ to $y$, then one says that $x$ is in the chain stable set of $y$. If $x$ is in the chain stable set of $y$ and $y$ is also in the chain stable set of $x$, then one says that $x$ and $y$ are chain related or chain equivalent. If $x$ is chain related with itself, then $x$ is called a chain recurrent point. Denote by $\mathrm{CR}(X)$ the set of chain recurrent points of $X$. Since chain related relation is a closed equivalent relation, one can divide $\mathrm{CR}(X)$ into closed equivalent classes. 
Each equivalent class is called a chain recurrent class. For $x \in \mathrm{CR}(X)$, we denote by $C(x)$ the chain recurrent class containing $x$. A chain recurrent class is called non-trivial if it does not reduce to a periodic orbit or a singularity.

To avoid some pathological phenomena, one may consider residual set of $\mathcal{X}^{r}(M)$. A subset $\mathcal{R} \subset \mathcal{X}^{r}(M)$ is residual if it contains a countable intersection of dense open subsets of $\mathcal{X}^{r}(M)$. Since $\mathcal{X}^{r}(M)$ is complete, every residual set is dense in $\mathcal{X}^{r}(M)$. We usually use the phrase "for $C^{1}$ generic $X$...", which means that "there exists a residual subset $\mathcal{R}$ such that for every $X \in \mathcal{R}$...".

\subsection{The precise statement of our results}

Our main result shows that, $C^{1}$-generically on 3-manifolds, the notions of singular hyperbolicity and dominated splitting coincide on chain recurrent classes containing a singular point and a regular periodic orbit:

Theorem A. Assume that $\operatorname{dim} M=3$. For $C^{1}$ generic $X \in \mathcal{X}^{1}(M)$, if the chain recurrent class $C(\sigma)$ of a singularity $\sigma$ contains a periodic orbit and admits $a$ dominated splitting $T_{C(\sigma)} M=E \oplus F$ with respect to $\Phi_{t}$, then $C(\sigma)$ is singular hyperbolic. Consequently, $C(\sigma)$ is an attractor or repeller according to the index of $\sigma$ equal to 2 or 1 .

If we do not assume that $C(\sigma)$ contains a periodic point, we get only a partially hyperbolic splitting:

Theorem B. Assume that $\operatorname{dim} M=3$. For $C^{1}$ generic $X \in \mathcal{X}^{1}(M)$, if the chain recurrent class $C(\sigma)$ of a singularity $\sigma$ is non-trivial and admits a dominated splitting $T_{C(\sigma)} M=E \oplus F$ with respect to $\Phi_{t}$, then $C(\sigma)$ is partially hyperbolic.

This result is closely related to the following conjecture of Morales and Pacifico ( [16]) in the spirit of conjectures of Palis ([18], [19, page 500, Conjecture 5]).

Conjecture 1.1. Given a closed 3 manifold, every vector field can be $C^{r}$ approximated by one of the following two kinds of vector fields:

- vector fields which are singular Axiom A without cycle,

- vector fields with a homoclinic tangency.

$X$ is called singular Axiom A without cycle if $X$ has only finitely many chain recurrent classes, and each chain recurrent class is singular hyperbolic. Singular Axiom A vector fields is a generalization of Axiom A vector fields. One says that $X$ has a homoclinic tangency if for some hyperbolic periodic orbit $\gamma$ of $X, W^{s}(\gamma)$ and $W^{u}(\gamma)$ have some non-transverse intersection.

Since we mainly consider 3 dimensional case in this paper, we will use $M^{3}$ to indicate the manifold $M$ is 3-dimensional. 


\section{Preliminaries}

\subsection{Dominated splittings}

As in the introduction, every vector field $X \in \mathcal{X}^{1}(M)$ generates a flow $\phi_{t}^{X}$. We identify the vector field and its generated flow as the same object. From the flow $\phi_{t}^{X}$, one can define its tangent flow $\Phi_{t}^{X}=\mathrm{d} \phi_{t}^{X}: T M \rightarrow T M$. For every regular point $x \in M \backslash \operatorname{Sing}(X)$, one can define its normal space

$$
\mathcal{N}_{x}=\left\{v \in T_{x} M:\langle v, X(x)\rangle=0\right\} .
$$

Define the normal bundle on regular points as:

$$
\mathcal{N}=\bigsqcup_{x \in M \backslash \operatorname{Sing}(X)} \mathcal{N}_{x} .
$$

On the normal bundle $\mathcal{N}$, one can define the linear Poincare flow $\psi_{t}^{X}$ : for each $v \in \mathcal{N}_{x}$,

$$
\psi_{t}(v)=\Phi_{t}(v)-\frac{\left\langle\Phi_{t}(v), X\left(\phi_{t}(x)\right)\right\rangle}{\left|X\left(\phi_{t}(x)\right)\right|^{2}} X\left(\phi_{t}(x)\right) .
$$

$\psi_{t}(v)$ is the orthogonal projection of $\Phi_{t}(v)$ on $\mathcal{N}$ along the flow direction. For an invariant (may be non-compact) set $\Lambda \subset M \backslash \operatorname{Sing}(X)$, one says that $\Lambda$ admits a dominated splitting with respect to the linear Poincare flow if there are constants $C \geq 1, \lambda>0$ and an invariant splitting $\mathcal{N}_{\Lambda}=\Delta^{s} \oplus \Delta^{u}$ such that for any $x \in \Lambda$ and $t \geq 0$, one has $\left\|\left.\psi_{t}\right|_{\Delta^{s}(x)}\right\|\left\|\left.\psi_{-t}\right|_{\Delta^{u}\left(\phi_{t}(x)\right)}\right\| \leq C \mathrm{e}^{-\lambda t}$. $\operatorname{dim} \Delta^{s}$ is called the index of the dominated splitting if it is a constant.

If $\Lambda$ is a non-singular compact invariant set, the existence of dominated splitting for the linear Poincare flow is a robust property.

Lemma 2.1 (5, page 288-289). Given $X \in \mathcal{X}^{1}(M)$, let $\Lambda$ be a non-singular compact invariant set of $X$. If $\Lambda$ admits a dominated splitting of index $i$ with respect to the linear Poincare flow, then there is $\varepsilon>0$ such that for each $Y$ which is $\varepsilon-C^{1}$ close to $X$, for any compact invariant set $\Lambda_{Y}$ contained in the $\varepsilon$ neighborhood of $\Lambda, \Lambda_{Y}$ admits a dominated splitting of index $i$ with respect to the linear Poincaré flow.

For dominated splittings with respect to tangent flows, no matter compact invariant sets contain singularity or not, we always have the robust property.

Lemma 2.2 (5, page 288-289). Given $X \in \mathcal{X}^{1}(M)$, let $\Lambda$ be a compact invariant set of $X$. If $\Lambda$ admits a dominated splitting of index $i$ with respect to the tangent flow, then for any $\varepsilon>0$, there exists $\delta>0$ such that for each $Y$ which is $\delta$ - $C^{1}$-close to $X$, for any compact invariant set $\Lambda_{Y}$ contained in the $\delta$ neighborhood of $\Lambda, \Lambda_{Y}$ admits a dominated splitting of index $i$ with respect to the tangent flow, which is $\varepsilon$ close to the dominated splitting of $\Lambda$. 
By the definition of linear Poincare flow, one has the following lemma immediately:

Lemma 2.3. Given $X \in \mathcal{X}^{1}(M)$, let $\Lambda$ be a compact invariant set of $X$. If $\Lambda$ admits a dominated splitting $T_{\Lambda} M=E \oplus F$ with respect to the tangent flow and $X(x) \in F(x)$ for any $x \in \Lambda$, then $\mathcal{N}_{\Lambda \backslash \operatorname{Sing}(X)}$ admits a dominated splitting of index $\operatorname{dim} E$ with respect to the linear Poincaré flow.

\subsection{Minimally non-hyperbolic set of $C^{2}$ vector fields}

When we discuss non-hyperbolic set, the non-hyperbolicity usually concentrates on some smaller parts, which are called minimally non-hyperbolic set $([12,15])$. Precisely, a compact invariant set $\Lambda$ is called minimally non-hyperbolic if $\Lambda$ is not hyperbolic and every compact invariant nonempty proper subset of $\Lambda$ is hyperbolic. From [3,22], one has the following two lemmas.

Lemma 2.4. Let $X \in \mathcal{X}^{1}\left(M^{3}\right)$ and $\Lambda$ a minimally non-hyperbolic set of $X$ with $\Lambda \cap \operatorname{Sing}(X)=\emptyset$. If $\mathcal{N}_{\Lambda}$ admits a dominated splitting with respect to the linear Poincaré flow, then $\Lambda$ is transitive.

Definition 2.5. $X$ is called weak Kupka-Smale if every periodic orbit and every singularity of $X$ are hyperbolic.

Lemma 2.6. Let $X \in \mathcal{X}^{2}\left(M^{3}\right)$ be weak Kupka-Smale and $\Lambda$ a minimally nonhyperbolic set of $X$ such that $\Lambda \cap \operatorname{Sing}(X)=\emptyset$. If $\mathcal{N}_{\Lambda}$ admits a dominated splitting with respect to the linear Poincaré flow, then $\Lambda$ is a normally hyperbolic torus and the dynamics on $\Lambda$ is equivalent to an irrational flow.

\subsection{Chain recurrence}

A compact invariant set $\Lambda$ is called chain transitive if for any $x, y \in \Lambda$ and any $\varepsilon>0$, there exists an $\varepsilon$-chain $\left\{\left(x_{0}, t_{0}\right),\left(x_{1}, t_{1}\right), \cdots,\left(x_{n}, t_{n}\right)\right\}$ from $x$ to $y$ such that $x_{i} \in \Lambda, i=0,1, \cdots, n$.

The following result is folklore for diffeomorphism case. We give here a proof for flows for the convenience of the reader.

Lemma 2.7. If $\Lambda$ is a non-trivial chain transitive set and contains a hyperbolic periodic orbit or a hyperbolic singularity $\gamma$, then $\Lambda \cap W^{s}(\gamma) \backslash\{\gamma\} \neq \emptyset$ and $\Lambda \cap$ $W^{u}(\gamma) \backslash\{\gamma\} \neq \emptyset$.

Proof. Since $\Lambda$ is non-trivial, there is $x_{0} \in \Lambda \backslash \gamma$. Since $\gamma$ is a hyperbolic periodic orbit or a hyperbolic singularity, it is isolated, i.e., there is an open neighborhood $U$ of $\gamma$ such that $x_{0} \notin \bar{U}$ and

$$
\gamma=\bigcap_{t \in \mathbb{R}} \phi_{t}(\bar{U})
$$

Take $V=\bigcap_{t \in[-2,2]} \phi_{t}(U)$ and fix a point $y \in \gamma$. Since $\Lambda$ is chain transitive, for every $n \in \mathbb{N}$, there is a $1 / n$-chain

$$
\left\{\left(x_{0, n}, t_{0, n}\right),\left(x_{1, n}, t_{1, n}\right), \cdots,\left(x_{k_{n}, n}, t_{k_{n}, n}\right)\right\}
$$


from $x_{0}$ to $y$ with $x_{i, n} \in \Lambda$. Choose $j_{n} \in\left[1, k_{n}\right]$ such that,

- $x_{j_{n-1}, n} \notin V$.

- $x_{i, n} \in V$ for $j_{n} \leq i \leq k_{n}$.

Let $z$ be an accumulation point of $\left\{x_{j_{n}, n}\right\}$. Then $z \in V \cap \Lambda$. Since $\phi_{[-2,0]}(z) \backslash$ $\operatorname{int}(V) \neq \emptyset, z \notin \gamma$. According to the second condition on $j_{n}$ and the definition of $V$, we have that $\phi_{t}(z) \in U$ for $t \geq 0$. Since $\gamma$ is isolated, $\omega(z)=\gamma$. Hence, $z \in W^{s}(\gamma) \cap \Lambda \backslash \gamma$.

The conclusion for $W^{u}(\gamma)$ can be proved similarly.

As a corollary of the above lemma, we have

Lemma 2.8. If $\Lambda$ is a non-trivial chain transitive set admitting a dominated splitting $T_{\Lambda} M=E \oplus F$ with respect to the tangent flow and $X(x) \in F(x)$ for any regular point $x \in \Lambda$, then for every hyperbolic singularity $\sigma \in \Lambda$, $\operatorname{ind}(\sigma)>\operatorname{dim} E$.

Proof. Suppose on the contrary that ind $(\sigma) \leq \operatorname{dim} E$ for some hyperbolic singularity $\sigma \in \Lambda$. Now, we have two dominated splittings at $\sigma$ :

$$
T_{\sigma} M=E(\sigma) \oplus F(\sigma), \quad \text { and } \quad T_{\sigma} M=E^{s} \oplus E^{u} .
$$

Since $\operatorname{ind}(\sigma) \leq \operatorname{dim} E$, i.e., $\operatorname{dim} E^{s} \leq \operatorname{dim} E(\sigma)$, according to [8, Lemma 3.5], one has $E^{s}(\sigma) \subset E(\sigma)$. By Lemma 2.7, there is $x \in W^{s}(\sigma) \cap \Lambda \backslash\{\sigma\}$. Thus, $X\left(\phi_{t}(x)\right) \subset T_{\phi_{t}(x)} W^{s}(\sigma)$ for any $t>0$. By the assumption one has $X\left(\phi_{t}(x)\right) \subset$ $F\left(\phi_{t}(x)\right)$. On the other hand, one has $\lim _{t \rightarrow+\infty}\left\langle X\left(\phi_{t}(x)\right)\right\rangle \subset E^{s}(\sigma) \subset E(\sigma)$. This fact contradicts to the continuity of dominated splittings.

For each compact set $K$ ( $K$ may not be invariant), one can define the chain recurrent set $\mathrm{CR}(X, K)$ in $K$. We say that $x \in \mathrm{CR}(X, K)$ if for any $\varepsilon>0$, there exists an $\varepsilon$-chain in $K$ from $x$ to $x$, i.e., there exists $\varepsilon$-chain $\left\{\left(x_{0}, t_{0}\right),\left(x_{1}, t_{1}\right), \cdots,\left(x_{n}, t_{n}\right)\right\}$ from $x$ to $x$ with $x_{i} \in K$ for $i=0,1, \cdots, n$. $\mathrm{CR}(X, K)$ has the following uppersemi continuity.

Lemma 2.9. Given $X \in \mathcal{X}^{1}(M)$ and a compact set $K$, if there are a sequence of $C^{1}$ vector fields $\left\{X_{n}\right\}$ and a sequence of compact sets $K_{n}$ such that

- $X_{n} \rightarrow X$ as $n \rightarrow \infty$ in the $C^{1}$ topology,

- $K_{n} \rightarrow K$ as $n \rightarrow \infty$ in the Hausdorff topology,

then $\lim \sup _{n \rightarrow \infty} \mathrm{CR}\left(X_{n}, K_{n}\right) \subset \mathrm{CR}(X, K)$.

Proof. Given a point $x \in \lim \sup _{n \rightarrow \infty} \mathrm{CR}\left(X_{n}, K_{n}\right)$, we will prove that $x \in \mathrm{CR}(X, K)$. First, there exists a sequence $x^{n} \in \mathrm{CR}\left(X_{n}, K_{n}\right)$ such that $\lim _{n \rightarrow \infty} x^{n}=x$.

According to the continuity of $\phi_{t}$, for any $\varepsilon>0$, there exists $\delta \in(0, \varepsilon / 4)$ such that if $d(x, y)<\delta$ then $d\left(\phi_{t}(x), \phi_{t}(y)\right)<\varepsilon / 4$ for $t \in[-2,2]$. Since 
$\lim _{n \rightarrow \infty} X_{n}=X$, for any $\varepsilon>0$, and $n$ large enough, for any $x \in M, t \in[-2,2]$, $d\left(\phi_{t}^{X_{n}}(x), \phi_{t}^{X}(x)\right)<\varepsilon / 4$. Assume that

$$
\left\{\left(x_{1}, t_{1}\right), \cdots,\left(x_{m}, t_{m}\right)\right\}
$$

is an $\varepsilon / 4$-chain in $K_{n}$ of $X_{n}$. Since $\lim _{n \rightarrow \infty} K_{n}=K$, for $n$ large enough, there exist $y_{i} \in K$ such that $d\left(x_{i}, y_{i}\right)<\delta$ for $i=1,2, \cdots, m$. We claim that

$$
\left\{\left(y_{1}, t_{1}\right), \cdots,\left(y_{m}, t_{m}\right)\right\}
$$

is an $\varepsilon$-chain in $K$ of $X$. In fact,

$$
\begin{aligned}
d\left(\phi_{t_{i}}\left(y_{i}\right), y_{i+1}\right) \leq & d\left(\phi_{t_{i}}\left(y_{i}\right), \phi_{t_{i}}\left(x_{i}\right)\right)+d\left(\phi_{t_{i}}\left(x_{i}\right), \phi_{t_{i}}^{X_{n}}\left(x_{i}\right)\right) \\
& +d\left(\phi_{t_{i}}^{X_{n}}\left(x_{i}\right), x_{i+1}\right)+d\left(x_{i+1}, y_{i+1}\right) \\
< & \varepsilon .
\end{aligned}
$$

So, given $\varepsilon>0$, for $n$ large enough, since there exists $\varepsilon / 4$-chain from $x^{n}$ to $x^{n}$ for $X_{n}$, we can get an $\varepsilon$-chain from $x$ to $x$ for $X$, which proves that $x \in \mathrm{C}(X, K)$.

By the upper-semi continuity, one has

Lemma 2.10. Given $X \in \mathcal{X}^{1}(M)$ and a compact set $K$, if $\mathrm{CR}(X, K)=\emptyset$, then there is a $C^{1}$ neighborhood $\mathcal{U}$ of $X$ and a neighborhood $U$ of $K$ such that $\operatorname{CR}(Y, \bar{U})=\emptyset$ for every $Y \in \mathcal{U}$.

Proof. If the conclusion is not true, there are a sequence of vector fields $\left\{X_{n}\right\}$ and a sequence of neighborhoods $\left\{U_{n}\right\}$ of $K$ such that

- $\lim _{n \rightarrow \infty} X_{n}=X$ and $\lim _{n \rightarrow \infty} \bar{U}_{n}=K$,

- $\mathrm{CR}\left(X_{n}, \bar{U}_{n}\right) \neq \emptyset$.

By Lemma 2.9, we have that $\mathrm{CR}(X, K) \neq \emptyset$ which contradicts the assumption. have

Combining the upper-semi continuity and robustness of hyperbolic set, we

Lemma 2.11. Given $X \in \mathcal{X}^{1}(M)$ and a compact set $K$, if $\mathrm{CR}(X, K)$ is hyperbolic, then there are a $C^{1}$ neighborhood $\mathcal{U}$ of $X$ and a neighborhood $U$ of $K$ such that $\mathrm{CR}(Y, \bar{U})$ is hyperbolic.

Proof. By the robustness of hyperbolicity, if $\mathrm{CR}(X, K)$ is a hyperbolic set of $X$, then there exist neighborhoods $\mathcal{U}_{1}$ of $X$ in $\mathcal{X}^{1}(M)$ and $U$ of $\operatorname{CR}(X, K)$ such that for any $Y \in \mathcal{U}_{1}$ and any compact invariant set $\Lambda \subset U$ of $Y, \Lambda$ is a hyperbolic set of $Y$.

By the upper semi-continuity of chain recurrence (Lemma 2.9), for the above neighborhood $U$ of $\mathrm{CR}(X, K)$, there is a $C^{1}$ neighborhood $\mathcal{U}_{2}$ of $X$, such that for any $Y \in \mathcal{U}_{2}, \mathrm{CR}(Y, \bar{U}) \subset U$.

Take $\mathcal{U}=\mathcal{U}_{1} \cap \mathcal{U}_{2}$. Then for any $Y \in \mathcal{U}, \mathrm{CR}(Y, \bar{U})$ is hyperbolic. 


\subsection{Ergodic closing lemma for flows}

We need the flow version [25] of Mañé's ergodic closing lemma [14].

Definition 2.12. $a \in M \backslash \operatorname{Sing}(X)$ is called strongly closable if for any $C^{1}$ neighborhood $\mathcal{U}$ of $X$, and any $\delta>0$, there are $Y \in \mathcal{U}$ and $p \in M, \pi(p)>0$ such that

- $\phi_{\pi(p)}^{Y}(p)=p$

- $X(x)=Y(x)$ for any $x \in M \backslash \bigcup_{t \in \mathbb{R}} B\left(\phi_{t}(a), \delta\right)$,

- $d\left(\phi_{t}^{X}(a), \phi_{t}^{Y}(p)\right)<\delta$ for each $t \in[0, \pi(p)]$.

Denote by $\Sigma(X)$ the set of strongly closable points of $X$.

Lemma 2.13 (Ergodic closing lemma for flows [25]). $\mu(\Sigma(X) \cup \operatorname{Sing}(X))=1$ for every $T>0$ and every $\phi_{T}^{X}$-invariant probability Borel measure $\mu$.

\subsection{Generic results}

We need the following generic results in this paper.

Lemma 2.14. There is a dense $G_{\delta}$ set $\mathcal{G} \subset \mathcal{X}^{1}(M)$ such that for each $X \in \mathcal{G}$, one has

1. Every periodic orbit and every singularity of $X$ are hyperbolic.

2. For any non-trivial chain recurrent class $C(\sigma)$, if $\sigma$ is a hyperbolic singularity of index $\operatorname{dim} M-1$, then $C(\sigma)$ is Lyapunov stable and every separatrix of $W^{u}(\sigma)$ is dense in $C(\sigma)$. Especially, $C(\sigma)$ is transitive.

3. Given $i \in[0, \operatorname{dim} M-1]$, if there is a sequence of vector fields $\left\{X_{n}\right\}$ such that

- $\lim _{n \rightarrow \infty} X_{n}=X$,

- each $X_{n}$ has a hyperbolic periodic orbits $\gamma_{n}$ of index $i$ such that $\lim _{n \rightarrow \infty} \gamma_{n}=$ $\Lambda$,

then $X$ itself has a sequence of hyperbolic periodic orbits $\gamma_{n}^{\prime}$ of index $i$ such that $\lim _{n \rightarrow \infty} \gamma_{n}^{\prime}=\Lambda$.

4. Every chain recurrent class $C(\sigma)$ is continuous with $X$ : for any $\varepsilon>0$, there is a neighborhood $\mathcal{U}$ of $X$ such that for any $Y \in \mathcal{U}$, one has $d_{H}\left(C\left(\sigma_{Y}\right), C(\sigma)\right)<\varepsilon$.

Remark 2.15. Item 1 is the classical Kupka-Smale theorem [11,23]. Item 2 is a corollary of the connecting lemma for pseudo-orbits [4]. [16, Section 4] gave some ideas about the proof of Item 2 without using of the terminology of chain recurrence. One can find the proof item 3 in [26] for diffeomorphism case. Item 4 holds because the upper semi-continuity of chain recurrent classes. 


\subsection{Saddle value of singularity}

Let $X \in \mathcal{X}^{r}\left(M^{3}\right)$ and $\sigma$ a hyperbolic singularity of $X$ of index 2. Assume that the three eigenvalues of $D X(\sigma)$ satisfy

$$
\operatorname{Re}\left(\lambda_{1}\right) \leq \operatorname{Re}\left(\lambda_{2}\right)<0<\lambda_{3} .
$$

$I(\sigma)=\operatorname{Re}\left(\lambda_{2}\right)+\lambda_{3}$ is called the saddle value of $\sigma$.

Lemma 2.16 ([27, page 207-219, Theorem 3.2.12]). Given $X \in \mathcal{X}^{1}\left(\mathbb{R}^{3}\right)$, assume that $\sigma$ is hyperbolic saddle and the three real eigenvalues of DX $(\sigma)$ satisfy

$$
\lambda_{1}<\lambda_{2}<0<\lambda_{3}<-\lambda_{2} .
$$

If one branch $W_{+}^{u}(\sigma)$ of the unstable manifold $W^{u}(\sigma)$ is a homoclinic orbit of $\sigma$, then for any $\varepsilon>0$, there exists $Y \in \mathcal{X}^{1}\left(\mathbb{R}^{3}\right)$ such that

$$
\sup \left\{|X(x)-Y(x)|,\|D X(x)-D Y(x)\|: x \in \mathbb{R}^{3}\right\}<\varepsilon,
$$

and $Y$ has a hyperbolic sink $\gamma_{Y}$. Furthermore, $W_{+}^{u}\left(\sigma_{Y}\right) \backslash\left\{\sigma_{Y}\right\}$ is contained in the attracting basin of $\gamma_{Y}$, where $\sigma_{Y}$ and $W_{+}^{u}\left(\sigma_{Y}\right)$ are the continuations of $\sigma$ and $W_{+}^{u}(\sigma)$

Remark 2.17. In Lemma 2.16, one can require that the support of $Y-X$ is in an arbitrarily small neighborhood of the homoclinic orbit.

By using the $C^{1}$ connecting lemma for pseudo-orbits [4], we have

Lemma 2.18. There exists a $C^{1}$ open dense subset $\mathcal{O} \subset \mathcal{X}^{1}\left(M^{3}\right)$ such that for any $X \in \mathcal{O}$, if $\sigma$ is a hyperbolic singularity and the three real eigenvalues of $D X(\sigma)$ satisfy

$$
\lambda_{1}<\lambda_{2}<0<\lambda_{3}<-\lambda_{2},
$$

then $C(\sigma)$ is trivial.

Proof. According to the upper-semi continuity of $\mathrm{CR}(X)$, if $C(\sigma)$ is trivial, then there exists a $C^{1}$ neighborhood $\mathcal{U}$ of $X$ such that for any $Y \in \mathcal{U}, C\left(\sigma_{Y}\right)$ is also trivial, where $\sigma_{Y}$ is the continuation of $\sigma$. So, to prove the lemma, we only have to show that for any $X \in \mathcal{X}^{1}\left(M^{3}\right)$, there exists an arbitrary small perturbation $Y$ such that if a singularity of $Y$ satisfies the assumption of the lemma, then its chain recurrent class is trivial.

After an arbitrary small perturbation, we may assume that every singularity of $X$ is hyperbolic. Consider a singularity $\sigma$ satisfying the assumption of the lemma. If $C(\sigma)$ is non-trivial, by using the $C^{1}$ connecting lemma, an arbitrary small perturbation $Y$ of $X$ has a homoclinic loop associated to the singularity $\sigma_{Y}$. According to Lemma 2.16, there exists arbitrary small perturbation $Z$ of $Y$ such that one branch $W_{+}^{u}\left(\sigma_{Z}\right)$ is attracted by a hyperbolic sink $\gamma_{Z}$. After another arbitrary small perturbation when necessary, we may assume that $Z$ satisfies the generic property of Lemma 2.14. And hence $C\left(\sigma_{Z}\right)$ is trivial. But this contradicts to Item 4 of Lemma 2.14. 
Since there exist only finitely many hyperbolic singularities, after finitely many arbitrary small perturbations, we will get a vector field whose singularities satisfying the assumption of the lemma all have the property: their chain recurrent class is trivial.

Note that [17] proved that singularities with the properties in Lemma 2.18 is disjoint from robustly transitive sets for three-dimensional flows.

\section{Lyapunov chain recurrent classes: Proof of Theorem A}

First, we get a $C^{1}$ generic property from the $C^{2}$ result of Lemma 2.6.

Lemma 3.1. For $C^{1}$ generic $X \in \mathcal{X}^{1}\left(M^{3}\right)$, if $\Lambda$ is non-singular chain transitive set and admits a dominated splitting $\mathcal{N}_{\Lambda}=\Delta^{s} \oplus \Delta^{u}$ with respect to $\psi_{t}$, then $\Lambda$ is hyperbolic.

Proof. Let $\left\{U_{n}\right\}$ be a countable basis of $M$ and $\mathcal{O}=\left\{O_{n}\right\}_{n \in \mathbb{N}}$ the family of finite union of $\left\{U_{n}\right\}$. For each $n$, define

$$
\begin{gathered}
\mathcal{H}_{n}=\left\{X \in \mathcal{X}^{1}(M): \operatorname{CR}\left(X, \bar{O}_{n}\right) \text { is hyperbolic or } \operatorname{CR}\left(X, \bar{O}_{n}\right)=\emptyset\right\}, \\
\mathcal{N}_{n}=\operatorname{Int}\left(\mathcal{X}^{1}(M) \backslash \mathcal{H}_{n}\right) .
\end{gathered}
$$

According to Lemma 2.11 and Lemma $2.10, \mathcal{H}_{n}$ is an open set. And hence $\mathcal{H}_{n} \cup \mathcal{N}_{n}$ is open and dense in $\mathcal{X}^{1}(M)$. Therefore,

$$
\mathcal{G}=\bigcap_{n \in \mathbb{N}}\left(\mathcal{H}_{n} \cup \mathcal{N}_{n}\right)
$$

is a dense $G_{\delta}$ set. We will prove that $\mathcal{G}$ satisfies the lemma. In fact, given $X \in \mathcal{G}$, let $\Lambda$ be a non-singular chain transitive set with a dominated splitting $\mathcal{N}_{\Lambda}=\Delta^{s} \oplus \Delta^{u}$ on the normal bundle $\mathcal{N}_{\Lambda}$ with respect to the linear Poincaré flow $\psi_{t}$. We will prove that $\Lambda=\operatorname{CR}(X, \Lambda)$ (since $\Lambda$ is chain transitive) is hyperbolic. Otherwise, by Lemma $2.4, \Lambda$ contains a minimally non-hyperbolic set $\Gamma$, which is transitive. Since $\Gamma$ is compact, for some $n \in \mathbb{N}, \Gamma \subset O_{n}$. According to Lemma 2.1, there is a $C^{1}$ neighborhood $\mathcal{U}$ of $X$ such that the maximal invariant set in $\bar{O}_{n}$ of $Y \in \mathcal{U}$ has a dominated splitting on the normal bundle with respect to $\psi_{t}^{Y}$

Since $\Gamma$ is not hyperbolic, one has $X \in \mathcal{N}_{n}$. Take a $C^{2}$ weak Kupka-Smale vector field $Y \in \mathcal{N}_{n} \cap \mathcal{U} . Y \in \mathcal{N}_{n}$ implies that $\operatorname{CR}\left(Y, \bar{O}_{n}\right)$ is not hyperbolic. On the other hand, $Y \in \mathcal{U}$ implies that the maximal invariant set in $\bar{O}_{n}$ of $Y$ has a dominated splitting on the normal bundle with respect to the linear Poincare flow. By Lemma 2.6, $\mathrm{CR}\left(Y, \bar{O}_{n}\right)=\Lambda_{1} \cup \Lambda_{2}$ with $\Lambda_{1} \cap \Lambda_{2}=\emptyset$, where $\Lambda_{1}$ is hyperbolic and $\Lambda_{2}=\cup_{1 \leq i \leq m} \mathbb{T}_{i}^{2}$ such that $\mathbb{T}_{i}^{2}$ is a normally hyperbolic torus, and the dynamics on $\mathbb{T}_{i}^{2}$ is equivalent to an irrational flow. Note that $\mathbb{T}_{i}^{2}$ is isolated in $\operatorname{CR}(Y)$. Fix a small neighborhood $U$ of $\Lambda_{2}$ so that $\operatorname{CR}(Y) \cap U=\Lambda_{2}$. 
Since Morse-Smale vector fields on $\mathbb{T}^{2}$ are dense, there is a small perturbation $Z \in \mathcal{N}_{n} \cap \mathcal{U}$ of $Y$ such that $Z(x)=Y(x)$ for $x \notin U, \phi_{t}^{Z} \mathbb{T}_{i}^{2}=\mathbb{T}_{i}^{2}$ and the dynamics on $\mathbb{T}_{i}^{2}$ of $Z$ is Morse-Smale. Hence, $\operatorname{CR}\left(Z, \bar{O}_{n}\right) \subset \Lambda_{1} \cup \Lambda_{2}$ is hyperbolic, which contradicts $Z \in \mathcal{N}_{n}$.

We need the following lemma to prove exponentially contracting/expanding properties:

Lemma 3.2. Let $\Lambda$ be a compact invariant set of $\phi_{t}$ and $f: \Lambda \rightarrow \mathbb{R}$ a continuous function. Fix $T>0$. If for any $x \in \Lambda$, there is $n(x) \in \mathbb{N}$ such that

$$
\sum_{i=0}^{n(x)-1} f\left(\phi_{i T}(x)\right)<0
$$

then there are constants $C \in \mathbb{R}$ and $\lambda>0$ such that for any $x \in \Lambda$ and any $n \in \mathbb{N}$, we have

$$
\sum_{i=0}^{n-1} f\left(\phi_{i T}(x)\right) \leq C-\lambda n
$$

Proof. By the continuity of the flow and the function, for any $x \in \Lambda$, there is a neighborhood $U(x)$ of $x$ and a number $c(x)>0$ such that for any $y \in U(x)$, we have

$$
\sum_{i=0}^{n(x)-1} f\left(\phi_{i T}(y)\right)<-c(x) .
$$

Because $\Lambda$ is compact, there are $x_{1}, x_{2}, \ldots, x_{m}$ in $\Lambda$ such that $\Lambda \subset \bigcup_{1 \leq i \leq m} U\left(x_{i}\right)$. Let $N=\max \left\{n\left(x_{1}\right), n\left(x_{2}\right), \cdots, n\left(x_{m}\right)\right\}$ and $\lambda=\min \left\{c\left(x_{1}\right), c\left(x_{2}\right), \cdots, c\left(x_{m}\right)\right\} / N$. Let

$$
C=\max _{x \in \Lambda, 1 \leq i \leq N}\left\{\sum_{j=0}^{i-1} f\left(\phi_{j T}(x)\right)\right\}+\lambda N
$$

Now for any $x \in \Lambda$ and any $n \in \mathbb{N}$, consider the orbit $\operatorname{arc} \phi_{[0, n T]}(x)$. Since $x \in \Lambda \subset \bigcup_{1 \leq i \leq n} U\left(x_{i}\right)$, we can fix a $k_{1} \in[0, m]$ such that $x \in U\left(x_{k_{1}}\right)$. Let $n_{1}=n\left(x_{k_{1}}\right)$. And then fix a $k_{2} \in[0, m]$ such that $\phi_{n\left(x_{k_{1}}\right) T}(x) \in U\left(x_{k_{2}}\right)$. Let $n_{2}=n_{1}+n\left(x_{k_{2}}\right)$. Inductively, we get a partition $0=n_{0}<n_{1}<n_{2}<\cdots<n_{l}=n$ such that $n_{j+1}-n_{j} \leq N$ for any $0 \leq j \leq l-1$ and

$$
\sum_{i=0}^{n_{j+1}-n_{j}-1} f\left(\phi_{i T}\left(\phi_{n_{j} T}(x)\right)\right) \leq-\lambda\left(n_{j+1}-n_{j}\right), \quad \forall 0 \leq j \leq l-2 .
$$

The above inequalities imply the conclusion.

By using Lemma 3.2, usually we take $f$ to be $\log \left\|\left.\Phi_{T}\right|_{E(x)}\right\|$ or $\log \left|\operatorname{Det}\left(\left.\Phi_{-T}\right|_{F(x)}\right)\right|$. 
Lemma 3.3. For $C^{1}$ generic $X \in \mathcal{X}^{1}\left(M^{3}\right)$, if a compact invariant set $\Lambda$ of $X$ has a dominated splitting $T_{\Lambda} M=E \oplus F$ of index 1 with respect to the tangent flow $\Phi_{t}$ such that

- There is $T>0$ such that for every singularity $\sigma \in \Lambda$, $\left|\operatorname{Det}\left(\left.\Phi_{T}\right|_{F(\sigma)}\right)\right|>1$.

- For every $x \in \Lambda \backslash \operatorname{Sing}(X),\langle X(x)\rangle \subset F(x)$.

- $F$ is not sectional expanding,

then there is a sequence of sinks $\left\{P_{n}\right\}$ such that $\lim _{n \rightarrow \infty} P_{n}=\Gamma \subset \Lambda$.

Proof. Assume that $X \in \mathcal{X}^{1}\left(M^{3}\right)$ has the generic properties in Lemma 2.14. If $\Lambda$ contains a periodic sink itself, then just take $P_{n}$ to be the periodic sink. If $\Lambda$ contains a periodic source $\gamma$, then $\gamma$ admits a hyperbolic splitting

$$
T_{\gamma} M=\langle X\rangle \oplus E^{u}, \quad \operatorname{dim} E^{u}=2 .
$$

By the assumptions, $T_{\Lambda}=E \oplus F$ is a dominated splitting w.r.t. the tangent flow, where $\operatorname{dim} F=2$. Thus, we have $E^{u}(\gamma)=F(\gamma)$. But this contradicts to the fact that $\langle X(x)\rangle \subset F(x)$ for any $x \in \Lambda \backslash \operatorname{Sing}(X)$.

From now on, we assume that $\Lambda$ contains neither periodic sink nor periodic source.

Define $\varphi(x)=\log \left|\operatorname{Det}\left(\left.\Phi_{-T}\right|_{F(x)}\right)\right|$ for each $x \in \Lambda$. We will prove this lemma by absurd. If for any $x \in \Lambda$, there is $n(x) \in \mathbb{N}$ such that

$$
\sum_{i=0}^{n(x)-1} \varphi\left(\phi_{-i T}(x)\right)<0,
$$

then by Lemma 3.2 (by considering the flow $\phi_{-t}$ ), there are $C$ and $\lambda>0$ such that for any $n \in \mathbb{N}$

$$
\begin{aligned}
\log \left|\operatorname{Det}\left(\Phi_{-n T} \mid F(x)\right)\right| & =\sum_{i=0}^{n-1} \log \left|\operatorname{Det}\left(\Phi_{-T} \mid F_{\left(\phi_{-i T}(x)\right)}\right)\right| \\
& =\sum_{i=0}^{n-1} \varphi\left(\phi_{-i T}(x)\right) \leq C-\lambda n .
\end{aligned}
$$

Thus, there is $C^{\prime}>0$ such that for any $t \geq 0$, one has $\mid \operatorname{Det}\left(\left.\Phi_{-t}\right|_{F(x)} \mid \leq C^{\prime} \mathrm{e}^{-\lambda t}\right.$. This will imply that $F$ is sectional expanding.

Since $F$ is not sectional expanding, there is $x \in \Lambda$ such that for any $n \in \mathbb{N}$, one has

$$
\frac{1}{n} \sum_{i=0}^{n-1} \varphi\left(\phi_{-i T}(x)\right) \geq 0 .
$$

Let $\delta_{x}$ be the Dirac atomic measure supported on $x$. Define

$$
v_{n}=\frac{1}{n} \sum_{i=0}^{n-1} \delta_{\phi_{-i T}(x)}
$$


Let $v$ be an accumulation point of $\left\{v_{n}\right\}_{n \in \mathbb{N}}$. Note that $v$ is an invariant measure with $\operatorname{supp}(v) \subset \Lambda$. Because $\varphi$ is a continuous function, one has

$$
\int \varphi d v \geq 0
$$

By using the ergodic decomposation theorem, there is an ergodic invariant measure $\mu$ with $\operatorname{supp}(\mu) \subset \Lambda$ such that

$$
\int \varphi d \mu \geq 0
$$

By Lemma 2.13, for the set of strongly closable set $\Sigma(X)$, one has $\mu(\Sigma(X) \cup$ $\operatorname{Sing}(X))=1$. Since for each singularity $\sigma \in \Lambda$ one has $\left|\operatorname{Det}\left(\Phi_{T} \mid F_{(\sigma)}\right)\right|>1$ by assumption, one gets $\mu(\Sigma(X))=1$. Since $\varphi$ is continuous, by Birkhoff ergodic theorem, for $\mu$ almost every point $x \in \operatorname{supp}(\mu) \cap \Sigma(X)$,

$$
\lim _{n \rightarrow \infty} \frac{1}{n} \sum_{i=0}^{n-1} \varphi\left(\phi_{-i T}(x)\right)=\int \varphi d \mu .
$$

We claim that $x$ is not periodic. Otherwise, since $X$ is $C^{1}$ generic, one may assume that $x$ is a hyperbolic periodic point. Since $\Lambda$ contains neither periodic sink nor periodic source, $x$ is a saddle. We assume that $\operatorname{Orb}(x)$ has the following hyperbolic splitting:

$$
T_{\operatorname{Orb}(x)} M=E^{s} \oplus\langle X\rangle \oplus E^{u} .
$$

Since $\operatorname{dim} F=2$, we have $F=\langle X\rangle \oplus E^{u}$. By the continuity and invariance of the splitting, for some constant $c$, we have $\angle\left(\langle X(y)\rangle, E^{u}(y)\right)>c$ for any $y \in \operatorname{Orb}(x)$. Thus, we have

$$
\left|\operatorname{Det}\left(\left.\Phi_{-n T}\right|_{F(x)}\right)\right|=\left\|\left.\Phi_{-n T}\right|_{E^{u}(x)}\right\|\left\|\left.\Phi_{-n T}\right|_{\langle X(x)\rangle}\right\| \sin \angle\left(E^{u}\left(\phi_{-n T}(x)\right), X\left(\phi_{-n T}(x)\right)\right)
$$

tends to zero exponentially. This implies

$$
\lim _{n \rightarrow \infty} \frac{1}{n} \sum_{i=0}^{n-1} \varphi\left(\phi_{-i T}(x)\right)<0 .
$$

The above inequality contradicts to

$$
\lim _{n \rightarrow \infty} \frac{1}{n} \sum_{i=0}^{n-1} \varphi\left(\phi_{-i T}(x)\right)=\int \varphi d \mu \geq 0 .
$$

Since $x$ is a strong closable point, for any $\varepsilon>0$ there are $Y$ which is $\varepsilon$ - $C^{1}$-close to $X$ and $p_{\varepsilon} \in M, \pi\left(p_{\varepsilon}\right)>0$ such that

- $\phi_{\pi\left(p_{\varepsilon}\right)}^{Y}\left(p_{\varepsilon}\right)=p_{\varepsilon}$,

- $d\left(\phi_{t}^{X}(x), \phi_{t}^{Y}\left(p_{\varepsilon}\right)\right)<\varepsilon$ for each $t \in\left[0, \pi\left(p_{\varepsilon}\right)\right]$. 
Since $x$ is non-periodic, one has $\pi\left(p_{\varepsilon}\right) \rightarrow \infty$ as $\varepsilon \rightarrow 0$. By the robustness of dominated splitting (Lemma 2.2), the $Y$-orbit of $p_{\varepsilon}$ also has a dominated splitting $E_{\varepsilon} \oplus F_{\varepsilon}$ and $F_{\varepsilon} \rightarrow F, E_{\varepsilon} \rightarrow E$ as $\varepsilon \rightarrow 0$ in the Grassman metric. As a corollary, one has

$$
\lim _{\varepsilon \rightarrow 0} \frac{1}{\left[\pi\left(p_{\varepsilon}\right) / T\right]} \sum_{i=0}^{n-1} \log \left|\operatorname{Det}\left(\left.D \Phi_{-T}^{Y}\right|_{F_{\varepsilon}\left(\phi_{-i T}^{Y}\left(p_{\varepsilon}\right)\right)}\right)\right| \geq 0 .
$$

Since the orbit of each periodic orbit has the dominated splitting, for each $p_{\varepsilon}$, the largest Lyapunov exponent along the orbit of $p_{\varepsilon}$ tends to zero as $\varepsilon \rightarrow 0$.

By Franks Lemma for flows $[6,14]$, since the largest Lyapunov exponent of $p_{\varepsilon}$ is arbitrarily close zero, after an arbitrarily small perturbation one can change the index of $\left\{\mathrm{Orb}\left(p_{\varepsilon}\right)\right\}$ to get a sink (see the proof in [7, Proposition 2.2 ] for details). As a corollary, there is a sequence of vector fields $\left\{X_{n}\right\}$ such that

- $\lim _{n \rightarrow \infty} X_{n}=X$ in the $C^{1}$ topology.

- Each $X_{n}$ has a sink $\gamma_{n}$ such that $\limsup _{n \rightarrow \infty} \gamma_{n} \subset \overline{\operatorname{Orb}(x)}$.

By taking subsequence when necessary, we may assume that $\lim _{n \rightarrow \infty} \gamma_{n}=\Gamma \subset$ $\overline{\operatorname{Orb}(x)}$. Since $X$ is $C^{1}$ generic, by item 3 of Lemma $2.14, X$ itself has a sequence of periodic sinks $\gamma_{n}^{\prime}$ such that $\lim _{n \rightarrow \infty} \gamma_{n}^{\prime}=\Gamma \subset \overline{\operatorname{Orb}(x)}$.

Now we will manage to prove Theorem B. Assume that we are under the assumptions of Theorem B. First we have

Lemma 3.4. Under the assumptions of Theorem B, we have

- either for every $x \in C(\sigma) \backslash \operatorname{Sing}(X), X(x) \in E(x)$,

- or for every $x \in C(\sigma) \backslash \operatorname{Sing}(X), X(x) \in F(x)$.

Proof. By Lemma 2.14, $C(\sigma)$ is transitive. We can take a point $a \in C(\sigma)$ such that $\omega(a)=C(\sigma)$.

If $X(a) \in E(a)$ or $X(a) \in F(a)$, then one can get the conclusion according to the invariance and continuity of dominated splitting. Now, assume $X(a) \notin E(a) \cup$ $F(a)$. Since $a \in \omega(a)$, there exists a sequence $t_{n} \rightarrow+\infty$ and $\phi_{t_{n}}(a) \rightarrow a$ as $n \rightarrow \infty$.

By the dominated property, one will have $\lim _{n \rightarrow \infty} \Phi_{t_{n}}(X(a)) \in F(a)$. Since $\Phi_{t_{n}}(X(a))=X\left(\phi_{t_{n}}(a)\right)$, one has $X(a)=\lim _{n \rightarrow \infty} X\left(\phi_{t_{n}}(a)\right)=\lim _{n \rightarrow \infty} \Phi_{t_{n}}(X(a)) \in$ $F(a)$, which gives a contradiction.

Corollary 3.5. If ind $(\sigma)=2$, then for any $y \in C(\sigma) \backslash \operatorname{Sing}(X), X(y) \in F(y)$. As a corollary, singularities in $C(\sigma)$ have the same index.

Proof. Now, we have two dominated splittings at $\sigma$ :

$$
T_{\sigma} M=E(\sigma) \oplus F(\sigma), \quad \text { and } \quad T_{\sigma} M=E^{s} \oplus E^{u} .
$$


Since ind $(\sigma)=2, \operatorname{dim} F(\sigma) \geq \operatorname{dim} E^{u}$, according to [8, Lemma 3.5], $F(\sigma) \supset E^{u}$. According to Lemma 2.7 and 2.14, $W^{u}(\sigma) \subset C(\sigma)$. Take a regular point $x \in$ $W^{u}(\sigma)$, then $X\left(\phi_{-t}(x)\right) \rightarrow E^{u} \subset F(\sigma)$ as $t \rightarrow+\infty$. By Lemma 3.4, one has $X\left(\phi_{-t}(x)\right) \subset F\left(\phi_{-t}(x)\right)$ for $t$ large enough. Using Lemma 3.4 again, we have that for any regular point $y \in C(\sigma), X(y) \in F(y)$.

If singularities in $C(\sigma)$ have different indices, then there are hyperbolic singularities $\sigma_{1}, \sigma_{2} \in C(\sigma)$ such that $\operatorname{ind}\left(\sigma_{1}\right)=1$ and $\operatorname{ind}\left(\sigma_{2}\right)=2$. Thus by previous arguments, for every $x \in C(\sigma) \backslash \operatorname{Sing}(X), X(x) \in E(x)$ and $X(x) \in F(x)$. This contradiction ends the proof.

Lemma 3.6. If $\operatorname{ind}(\sigma)=2$, then $\operatorname{dim} E=1$ and $E$ is contracting.

Proof. First by Corollary 3.5, for every $x \in C(\sigma) \backslash \operatorname{Sing}(X), X(x) \in F(x)$. According to Lemma 2.7, $C(\sigma) \cap W^{s}(\sigma) \backslash\{\sigma\} \neq \emptyset$. This implies that the stable subspace $E^{s}$ of hyperbolic splitting at $\sigma$ has non trivial intersection with $F(\sigma)$. Hence $\operatorname{dim} F=2$ and then $\operatorname{dim} E=1$.

We will prove that $E$ is contracting. According to Corollary 3.5, every singularity $\sigma^{\prime}$ in $C(\sigma)$ has index 2 and $E\left(\sigma^{\prime}\right) \subset E^{s}\left(\sigma^{\prime}\right)$. For any point $x \in \Sigma$, there are two cases:

1. $\omega(x) \subset \operatorname{Sing}(X)$,

2. $\omega(x) \backslash \operatorname{Sing}(X) \neq \emptyset$.

In the first case, there is $t>0$ such that $\left\|\left.\Phi_{t}\right|_{E(x)}\right\|<1$. In the second case, take $y \in \omega(x) \backslash \operatorname{Sing}(X)$ and a small neighborhood $U$ of $y$ such that for any $y_{1}, y_{2} \in U$,

$$
\frac{1}{2} \leq \frac{\left|X\left(y_{1}\right)\right|}{\left|X\left(y_{2}\right)\right|} \leq 2 .
$$

Since $y \in \omega(x)$, there exists a sequence $t_{n} \rightarrow+\infty$ such that $\phi_{t_{n}}(x) \rightarrow y$ as $n \rightarrow \infty$. So, we may assume that $\phi_{t_{n}}(x) \in U$ for all $n$. Thus,

$$
\frac{\left|X\left(\phi_{t_{n}}(x)\right)\right|}{|X(x)|}=\frac{\left|X\left(\phi_{t_{1}}(x)\right)\right|}{|X(x)|} \frac{\left|X\left(\phi_{t_{n}}(x)\right)\right|}{\left|X\left(\phi_{t_{1}}(x)\right)\right|} \leq 2 \frac{\left|X\left(\phi_{t_{1}}(x)\right)\right|}{|X(x)|} .
$$

Since $E \oplus F$ is a dominated splitting and $X \in F$, there are constants $\lambda>0$ and $C \geq 1$ such that

$$
\left\|\Phi_{t_{n}} \mid E(x)\right\| \leq C \mathrm{e}^{-\lambda t_{n}} \frac{\left|X\left(\phi_{t_{n}}(x)\right)\right|}{|X(x)|} \leq 2 C \mathrm{e}^{-\lambda t_{n}} \frac{\left|X\left(\phi_{t_{1}}(x)\right)\right|}{|X(x)|} .
$$

When $n$ is large enough, one has $\left\|\Phi_{t_{n}} \mid E(x)\right\|<1$

So, for any $x \in C(\sigma)$, there is $t>0$ such that $\left\|\left.\Phi_{t}\right|_{E(x)}\right\|<1$. By Lemma 3.2, $E$ is uniformly contracting.

Since $\operatorname{dim} M=3$, every hyperbolic singularity in a non-trivial chain recurrent class is either index 1 or index 2, Lemma 3.6 ends the proof of Theorem B.

We will manage to prove Theorem A now. 
Proof of Theorem A. We assume that $X \in \mathcal{X}^{1}\left(M^{3}\right)$ has all the generic properties in Lemma 2.14, Lemma 2.18, Lemma 3.1 and Lemma 3.3.

Without loss of generality, we assume that ind $(\sigma)=2$. By Lemma 2.18, we have $I(\sigma)>0$ since $C(\sigma)$ is non-trivial. By Lemma 3.6, the dominated splitting over $C(\sigma)$ is a partially hyperbolic splitting $T_{C(\sigma)} M=E^{s s} \oplus F$ with $\operatorname{dim} E^{s s}=1$.

Suppose on the contrary that $F$ is not sectional expanding. By Lemma 3.3, there is a sequence of sinks $\left\{P_{n}\right\}$ of $X$ such that $\lim _{n \rightarrow \infty} P_{n}=\Lambda \subset C(\sigma)$.

We claim that $\Lambda \cap \operatorname{Sing}(X) \neq \emptyset$. In fact, otherwise, by Lemma $3.1, \Lambda$ is a hyperbolic set in $C(\sigma)$, which could not be approximated by periodic sinks $P_{n}$.

Now take a singularity $\sigma^{\prime} \in \Lambda$. According to Corollary 3.5 , ind $\left(\sigma^{\prime}\right)=2$. And Lemma 2.7 tells us that $\Lambda \cap W^{u}\left(\sigma^{\prime}\right) \backslash\left\{\sigma^{\prime}\right\} \neq \emptyset$. By Lemma 2.14, every separatrix of $W^{u}\left(\sigma^{\prime}\right)$ is dense in $C(\sigma)$. Hence, $\Lambda=C(\sigma)$. By the assumption of this theorem, $\Lambda$ contains the hyperbolic periodic point $p$. Thus, there are $p_{n} \in P_{n}$ such that $\lim _{n \rightarrow \infty} p_{n}=p$. Since $C(\sigma)$ is Lyapunov stable (by Lemma 2.14), $W_{l o c}^{u}(\operatorname{Orb}(p)) \subset C(\sigma)$. So, for $n$ large enough, $W_{l o c}^{s s}\left(p_{n}\right) \cap W_{l o c}^{u}(\operatorname{Orb}(p)) \neq \varnothing$, which implies $p_{n} \in C(\sigma)$. This contradiction proves that $F$ is sectional expanding.

Finally, according to Theorem $\mathrm{D}$ in [16], $C(\sigma)$ is an attractor. This finishes the proof of Theorem A.

\section{References}

[1] V. AFRAM̆OVIČ, V. BYKOV and L. SiLniKOV, The origin and structure of the Lorenz, attractor, Dokl. Akad. Nauk SSSR 234 (1977), 336-339.

[2] V. Araujo and M. PACIFICO, "Three-dimensional Flows", Springer-Verlag, 2010.

[3] A. Arroyo and F. Rodriguez Hertz, Homoclinic bifurcations and uniform hyperbolicity for threedimensional flows, Ann. Inst. H. Poincaré Anal. Non Linéaire 20 (2003), 805841.

[4] C. Bonatti and S. Crovisier, Récurrence et généricité, Invent. Math., 158 (2004), 33104.

[5] C. Bonatti L. Díaz and M. Viana, "Dynamics Beyond Uniform Hyperbolicity. A Global Geometric and Probabilistic Perspective", Encyclopaedia of Mathematical Sciences, Vol. 102, Mathematical Physics, III, Springer-Verlag, Berlin, 2005, xviii+384 pp.

[6] C. BonatTi, N. Gourmelon and T. VIVIER, Perturbations of the derivative along periodic orbits, Ergodic Theory Dynam. Systems 26 (2006), 1307-1337.

[7] S. GAN, Horseshoe and entropy for $C^{1}$ surface diffeomorphisms, Nonlinearity 15 (2002), $1-8$.

[8] S. GAN and L. WEN, Nonsingular star flows satisfy axiom A and the no-cycle condition, Invent. Math. 164 (2006), 279-315.

[9] J. GUCHENHEIMER, A strange, strange attractor, The Hopf bifurcation theorems and its applications (Applied Mathematical Series), 19, Springer-Verlag, 1976, 368-381.

[10] J. GUCHENHEIMER and R. Williams, Structural stability of Lorenz attractors, Inst. Hautes Études Sci. Publ. Math. 50 (1979), 59-72.

[11] I. KuPKA, Contribution à la théorie des champs génériques, Contrib. Differ. Equations 2 (1963), 457-484 and 3 (1964), 411-420.

[12] S. LiaO, Obstruction sets II, Acta Sci. Natur. Univ. Pekinensis, 2 (1981), 1-36.

[13] E. N. LoREnZ, Deterministic nonperiodic flow, J. Atmosph. Sci. 20 (1963), 130-141.

[14] R. MAÑé, An ergodic closing lemma, Ann. of Math., 116 (1982), 503-540. 
[15] R. MAÑ́é, Hyperbolicity, sinks and measure in one-dimensional dynamics, Comm. Math. Phys. 100 (1985), 495-524.

[16] C. MORALES and M. PACIFICO, A dichotomy for three-dimensional vector fields, Ergodic Theory Dynam. Systems 23 (2003), 1575-1600.

[17] C. Morales, M. PACIFICO and E. PujALs, Robust transitive singular sets for 3-flows are partially hyperbolic attractors or repellers, Ann. of Math. 160 (2004), 375-432.

[18] J. PALIS, A global view of dynamics and a conjecture of the denseness of finitude of attractors, Astérisque 261 (2000), 335-347.

[19] J. PALIS, A global perspective for non-conservative dynamics, Ann. Inst. H. Poincaré Anal. Non Linéaire 22 (2005), 485-507.

[20] J. PALIS, Open questions leading to a global perspective in dynamics, Nonlinearity 21 (2008), 37-43.

[21] M. M. Регхото, Structural stability on two-dimensional manifolds, Topology 1 (1962), 101-120.

[22] E. PUJALS and M. S AMBARINO, Homoclinic tangencies and hyperbolicity for surface diffeomorphisms, Ann. of Math. 151 (2000), 961-1023.

[23] S. Smale, Stable manifolds for differential equations and diffeomorphisms, Ann. Sc. Norm. Super. Pisa Cl. Sci. 17 (1963), 97-116.

[24] S. SMALE, Diffeomorphisms with many periodic points, In: "Differential and Combinatorial Topology", Princeton Math. Ser. 24, Princeton University Press (1965), 63-68 ( ).

[25] L. WEN, On the $C^{1}$-stability conjecture for flows, J. Differential Equations 129 (1996), 334-357.

[26] L. WEN, Generic diffeomorphisms away from homoclinic tangencies and heterodimensional cycles, Bull. Braz. Math. Soc. (N.S.) 35 (2004), 419-452.

[27] S. Wiggins, "Global Bifurcations and Chaos-Analytical Methods", Springer-Verlag, 1988.

Institut de Mathématiques de Bourgogne

Université de Bourgogne(Dijon)

Dijon, 21004, France

bonatti@u-bourgogne.fr

School of the Mathematical Sciences

Peking University

Beijing 100871, P. R. China

gansb@math.pku.edu.cn

School of Mathematical Sciences

Soochow University

Suzhou, 215006, P. R. China

yangdw@suda.edu.cn 